\title{
La vaccinazione dell'epatite $B$ nei pazienti con insufficienza renale cronica
}

\section{Franco Giovanetti}

\author{
Dipartimento di Prevenzione ASL CN2 Alba Bra, Regione Piemonte, Alba (CN)
}

\begin{abstract}
HePATITIS B IMMUNIZATION IN PATIENTS WITH CHRONIC RENAL FAILURE
Abstract. Despite the control measures, such as hygiene precautions and segregation of infectious patients in dialysis units as well as immunization of susceptible patients, it has been estimated that in developed countries the prevalence of chronic carriers of the hepatitis B virus among hemodialysis patients is, on average, $3 \%$. An important cause of this unsatisfactory control of $\mathrm{HBV}$ infection in hemodialysis units is the suboptimal response to $\mathrm{HBV}$ vaccines among chronic kidney failure patients. This concise review highlights the common issues regarding vaccination against hepatitis $B$ in patients on hemodialysis and presents the currently available solutions.
\end{abstract}

Key words: Chronic renal failure, Hemodialysis, Immunization, Hepatitis B

Conflict of interest: The Author accepted travel grants from Pfizer, Novartis, Sanofi Pasteur, and GlaxoSmithKline for scientific meetings.

Financial support: None.

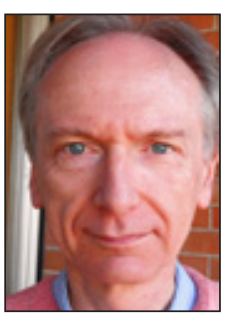

Franco Giovanetti
Nonostante l'adozione di misure di controllo, come le precauzioni igieniche e la segregazione dei pazienti HBsAg positivi nelle unità di dialisi, nonché l'immunizzazione dei suscettibili, si stima che, nei paesi sviluppati, la prevalenza dei portatori del virus dell'epatite $\mathrm{B}(\mathrm{HBV})$ tra $\mathrm{i}$ pazienti in emodialisi sia in media del 3\% (1).

L'insufficienza renale cronica (IRC) induce una depressione dell'immunità umorale e cellulare. L'alterazione più importante è rappresentata da una difettosa stimolazione dei linfociti $\mathrm{T}$ da parte delle cellule che presentano l'antigene (2). Questo fatto si traduce in una minore risposta ai vaccini ed è stato osservato dopo la somministrazione sia del tossoide tetanico e difterico che del vaccino antiepatite $\mathrm{B}(2,3)$. Inoltre è stata osservata una diminuita attivazione della memoria immunologica dopo stimolazione antigenica (4). Ne consegue che, non potendo fondarsi sulla memoria immunologica, la protezione del paziente con IRC verso l'infezione da virus dell'epatite B (HBV) dipende essenzialmente dalla concentrazione nel siero degli anticorpi neutralizzanti (anti-HBs) indotti dalla somministrazione dei vaccini attualmente disponibili, le cui caratteristiche principali sono riportate nella Tabella I.

La concomitante infezione da virus dell'epatite $\mathrm{Ce}$, in generale, la presenza di un'epatopatia cronica sembrano essere ulteriori cause di una diminuita risposta al vaccino antiepatite B (5). Altre cause di risposta insoddisfacente sono l'età avanzata, l'obesità, la presenza di una malattia celiaca non diagnosticata, il fumo di tabacco e la somministrazione del vaccino $\mathrm{HBV}$ nel gluteo anziché nel muscolo deltoide (6).

Fino a tempi recenti, sono state essenzialmente due le vie seguite per migliorare la risposta immune nei pazienti con IRC: a) l'uso di protocolli che prevedono un maggior numero di dosi o dosaggi più elevati di antigene;

b) la somministrazione intradermica dell'antigene.

Riguardo al primo punto, gli studi effettuati sugli emodializzati hanno riportato percentuali di sieroconversione differenti a seconda della schedula e del dosaggio utilizzati: 3 dosi della formulazione da $20 \mathrm{mcg}$ a 0-1-6 mesi hanno determinato la sieroconversione nel 39\% dei vaccinati (7), mentre l'impiego della dose da 40 mcg o di una schedula di 4 dosi (0-1-2-6 mesi) ha portato il tasso di sieroconversione al $60-86 \%$ a seconda dello studio (8-14).

Uno studio che ha utilizzato il vaccino da $10 \mathrm{mcg}$ con tre doppie dosi (quindi $20 \mathrm{mcg} / \mathrm{dose}$ ) ai tempi 0-1-6 mesi ha evidenziato un tasso di sieroconversione dell' $80 \%$, superiore a quello ottenuto con le usuali tre dosi da $10 \mathrm{mcg}$; tale studio evidenziava anche una migliore risposta nei soggetti sottoposti a dialisi peritoneale rispetto agli emodializzati (15).

In merito alla somministrazione intradermica, è stata osservata una migliore risposta anti-HBs al termine del ciclo vaccinale, ma non sono evidenti differenze significative a lungo termine. Inoltre la somministrazione intradermica appare tecnicamente più difficoltosa ed è stata osservata un'aumentata incidenza di 
TABELLA I - CARATTERISTICHE DEI VACCINI CONTRO L'EPATITE B ATTUALMENTE DISPONIBILI

\begin{tabular}{|c|c|c|c|c|}
\hline Dosaggio in microgrammi & Età di somministrazione & Adiuvante & Quantità di Al & Calendari vaccinali \\
\hline 5 & $0-15$ anni & \multirow{3}{*}{$\begin{array}{l}\text { Idrossifosfato amorfo di } \\
\text { Al solfato }\end{array}$} & $0.25 \mathrm{mg}$ & 0-1-6 mesi/0-1-2-12 mesi \\
\hline 10 & $>=16$ anni & & $0.50 \mathrm{mg}$ & 0-1-6 mesi/0-1-2-12 mesi \\
\hline 40 & Adulti (predialisi o dialisi) & & $0.50 \mathrm{mg}$ & 0-1-6 mesi \\
\hline 10 & $0-15$ anni & \multirow[t]{2}{*}{ Idrossido di Al } & $0.25 \mathrm{mg}$ & 0-1-6 mesi/0-1-2-12 mesi \\
\hline 20 & $>=16$ anni & & $0.50 \mathrm{mg}$ & 0-1-6 mesi/0-1-2-12 mesi \\
\hline 20 & $>=15$ anni (predialisi o dialisi) & AS04 (MPL + fosfato di Al) & $0.50 \mathrm{mg}$ & $0-1-2-6$ mesi \\
\hline
\end{tabular}

reazioni locali $(16,17)$.

Un'altra modalità per migliorare la risposta immune riguarda l'epoca della vaccinazione: la somministrazione del vaccino prima che si renda necessario il trattamento dialitico è seguita da un più alto tasso di sieroconversione e da più elevati titoli anticorpali (18).

Poiché l'incremento del dosaggio di antigene e del numero di dosi somministrate ha dimostrato di migliorare la risposta rispetto alla schedula standard senza, tuttavia, risolvere il problema dei non responder e degli ipo-responder, i ricercatori hanno rivolto la loro attenzione verso gli adiuvanti. L'adiuvante a base di sali di alluminio, comunemente utilizzato nell'antiepatite $\mathrm{B}$ così come in molti altri vaccini inattivati, appare inadeguato nell'indurre una soddisfacente risposta immunitaria nei pazienti con IRC. Tra i numerosi adiuvanti sperimentati, l'unico che sinora ha trovato un'applicazione pratica in questo ambito è stato $\mathrm{AS} 04$, un mono-fosforil-lipide$A$ (MPL) adsorbito in alluminio. Si tratta della combinazione di un sale di alluminio con il fosforil-lipide A di Salmonella minnesota. La sua azione consiste nell'attivazione del Tolllike receptor 4 delle cellule presentanti l'antigene. La contemporanea presenza dell'antigene di superficie dell'HBV (che rappresenta l'antigene vaccinale) e del fosforil-lipide $A$ induce una forte risposta immunitaria, che include sia l'immunità umorale che quella cellulare; i risultati sono una valida risposta anticorpale (anti-HBs) e la formazione di cellule della memoria immunitaria, la cui presenza è associata alla protezione a lungo termine. AS04 è presente anche nel vaccino bivalente contro il Papillomavirus.

Il vaccino adiuvato con AS04 è registrato per l'immunizzazione dei pazienti con IRC in pre-emodialisi ed emodialisi, a partire dai 15 anni di età, e prevede 4 dosi da somministrare nei tempi 0-1-2-6 mesi. Uno studio clinico ha dimostrato che i pazienti con IRC vaccinati utilizzando il vaccino adiuvato con $\mathrm{AS} 04$ presentano un più elevato tasso di persistenza nel tempo degli anticorpi protettivi, una più elevata percentuale di elevato titolo anticorpale $(>=100 \mathrm{mUI} / \mathrm{mL})$ e una migliore risposta dopo una dose booster rispetto ai soggetti che hanno ricevuto il vaccino convenzionale (19). Il profilo di sicurezza del vaccino è del tutto soddisfacente: sono state riportate transitorie reazioni locali, soprattutto dolore nel sito di iniezione, e generali (astenia, disturbi gastroenterici e cefalea) (20). In conclusione, il vaccino adiuvato con AS04 trova la sua indi- cazione nel priming dei soggetti in pre-emodialisi ed emodialisi, ma può essere utilizzato anche per i richiami nei pazienti che hanno già effettuato un ciclo primario con altri tipi di vaccino e il cui titolo anti-HBs sia sceso al di sotto dei $10 \mathrm{mUL} / \mathrm{mL}$, la soglia universalmente considerata protettiva.

\section{Riassunto}

Nonostante le misure di controllo, come le precauzioni igieniche e la segregazione dei pazienti HBsAg positivi nelle unità di dialisi, nonché l'immunizzazione dei suscettibili, si stima che, nei paesi sviluppati, la prevalenza dei portatori del virus dell'epatite $B$ tra i pazienti in emodialisi sia in media del $3 \%$. Una ragione importante per il controllo insoddisfacente delle infezioni da HBV nelle unità di emodialisi è la risposta sub-ottimale ai vaccini HBV tra i pazienti con insufficienza renale cronica. Questa breve review mette in evidenza i problemi comuni riguardanti la vaccinazione contro l'epatite $\mathrm{B}$ dei pazienti in emodialisi e presenta le soluzioni attualmente disponibili.

Parole chiave: Insufficienza renale cronica, Emodialisi, Vaccinazione, Epatite B

Dichiarazione di conflitto di interessi: L'Autore ha accettato inviti da parte di aziende produttrici di vaccini (Glaxo Smith Kline, Sanofi Pasteur, Pfizer) per la partecipazione a convegni scientifici.

Contributi economici agli Autori: L'Autore dichiara di non aver ricevuto sponsorizzazioni economiche per la preparazione dell'articolo.
Indirizzo dell'Autore:
Dr. Franco Giovanetti
Dipartimento di Prevenzione
ASL CN2 Alba Bra
Regione Piemonte
Via Vida 10
12051 Alba (CN)
fgiovanetti@aslcn2.it 


\section{Bibliografia}

1. Burdick RA, Bragg-Gresham JL, Woods JD, et al. Patterns of hepatitis B prevalence and seroconversion in hemodialysis units from three continents: The DOPPS. Kidney Int 2003; 63: 2222-9.

2. Krueger S, Seyfarth M, Sack K, Keeft B. Defective immune response to tetanus toxoid in hemodialysis patients and its association with diphtheria vaccination. Vaccine 1999; 17: $1145-50$.

3. Kreft B, Klouche M, Kreft R, Kirchner H, Sack K. Low efficiency of active immunization against diphtheria in chronic hemodialysis patients. Kidney Int 1997; 52: 212-6.

4. Litjens NH, Huisman M, van den Dorpel M, Betjes MG. Impaired immune responses and antigen-specific memory CD4+ T cells in hemodialysis patients. J Am Soc Nephrol 2008; 8: 1483-90.

5. Navarro JF, Teruel JL, Mateos ML, Ortuno J. Antibody level after hepatitis $\mathrm{B}$ vaccination in hemodialysis patients: influence of hepatitis $\mathrm{C}$ virus infection. Am J Nephrol 1996; 16: 95-7.

6. Wood RC, MacDonald K, White KE, et al. Risk factors for lack of detectable antibody following hepatitis B vaccination of Minnesota health care workers. JAMA 1993; 270: 2935-9.

7. Fleming SJ, Moran DM, Cooksley WGE, Faoagali JI. Poor immune response to a recombinant hepatitis $\mathrm{B}$ vaccine in dialysis patients. J Infect 1991; 22: 251-7.

8. Bergia R, Pellerey M, Berto I, et al. Hepatitis B vaccination in uremic patients: comparison between recombinant and plasma derived vaccine. Nephron 1992; 61: 328.

9. Bruguera M, Cremades M, Rodicio JL, et al. Immunogenicity of a yeast-derived hepatitis B vaccine in hemodialysis patients. Am J Med 1989; 87: S30-2.
10. Buti M, Viladomiu L, Jardi R, et al. Long term immunogenicity and efficacy of hepatitis $\mathrm{B}$ vaccine in hemodialysis patients. Am J Nephrol 1992; 12: 144-7.

11. Dentico P, Volpe A, Buongiorno R, et al. Immunogenicity and efficacy of anti-hepatitis B vaccines in hemodialysis patients. Nephron 1992; 61: 324-5.

12. Docci C, Cipolloni PA, Mengozzi S, et al. Immunogenicity of a recombinant DNA hepatitis B vaccine in patients with chronic renal failure. Epidemiol News Bull 1989; 15: 45-6.

13. Guan R, Choong L. Immunogenicity of a recombinant DNA hepatitis B vaccine in patients with chronic renal failure. Epidemiol News Bull 1989; 15: 45-6.

14. Marangi AL, Giordano R, Montanaro A, et al. Hepatitis B virus infection in chronic uremia: long term follow up of a two-step integrated protocol of vaccination. Am J Kidney Dis 1994; 23: 534-42.

15. Mitwalli A. Responsiveness to hepatitis B vaccine in immunocompromised patients by doubling the dose scheduling. Nephron 1996; 73: 417-20.

16. Fabrizi F, Dixit V, Magnini M, Elli A, Martin P. Metaanalysis: intradermal vs. intramuscular vaccination against hepatitis B virus in patients with chronic kidney disease. Aliment Pharmacol Ther 2006; 24: 497-506.

17. Chau KF, Cheng YL, Tsang DN, et al. Efficacy and side effects of intradermal hepatitis $B$ vaccination in CAPD patients: a comparison with the intramuscular vaccination. Am J Kidney Dis 2004; 43: 910-7.

18. Seaworth B, Drucker J, Starling J, Drucker R, Stevens C, Hamilton J. Hepatitis B vaccines in patients with chronic renal failure before dialysis. J Infect Dis 1988; 157 (2): 322-7.

19. Kong NC, Beran J, Kee SA, et al. A new adjuvant improves the immune response to $\mathrm{HB}$ vaccine in hemodialysis patients. Kidney Int 2008; 73: 856-62.

20. Kundi M. New hepatitis B vaccine formulated with an improved adjuvant system. Expert Rev Vaccines 2007; 2: 133-40. 